\title{
Communication Assistant for Deaf, Dumb and Blind
}

\author{
Rajyashree, O. Deepak, Naresh Rengaswamy, \\ K.S. Vishal
}

\begin{abstract}
Tending to the issues of people with vocal, visual and hearing troubles through a solitary helping framework is a difficult task. Numerous researches focus on tending to the issues of one of the above difficulties yet not all. Thanks to technology, this paper focuses on achieving the best technique that helps the visually impaired by letting them listen what is represented as text as well as feel it in braille which is achieved using a device that can understand the image and converts the content and present it as sounds. Using the speech to text conversion technique, on-screen text provides a better way for the people with Hearing impairment to visually read which is in audio. The vocally impaired people can interact with normal people using our work which recognizes sign languages and converts them into on-screen text as well as audio sounds. This single unique system powered by Raspberry Pi is designed to support all these three solutions.
\end{abstract}

Keywords--- Deaf, Dumb, Blind, Braille Device, Python Tesseract, Bluetooth, Speech to Text, Text to Speech.

\section{INTRODUCTION}

Having difficulties like being visually impaired, hard of hearing, dumb are a greater amount of concern. Science and innovation have influenced people to turn out to be dependent on solace yet there exists an underprivileged gathering of individuals who are battling for finding a creative way that can make the procedure of communication simpler for them. As indicated by the World Health Organization, around 285 million individuals on the planet are visually impaired, 300 million are hard of hearing and 1 million are mute[1]. In everyday life communication is a serious issue for the deaf, dumb and blind people.

The main correspondence channel accessible for people with hearing disability, visually impaired individuals is the tactile channel. Hence, technological assistive gadgets for hard of hearing, visually impaired individuals should some way convert internal stimuli into useful tactile sensations. [2]. There are many factors hindering the developments of such assistive devices like the deaf-blind population is very low. This speaks to a restricted market and restrictive expenses.

In this paper, a portable communication aid is designed which is used to communicate between disabled and normal people. The gestures created by the disabled person will be

\footnotetext{
Manuscript received September 16, 2019.

Rajyashree, Assistant Professor, Computer Science Engineering, SRM Institute of Science and Technology, Tamil Nadu, India. (e-mail: rrajyashree123@gmail.com)

O. Deepak, Student, Computer Science Engineering, SRM Institute of Science and Technology, Tamil Nadu, India. (e-mail: call.me.od274@gmail.com)

Naresh Rengaswamy, Student, Computer Science Engineering, SRM Institute of Science and Technology, Tamil Nadu, India. (e-mail: naresh.rengsawamy@gmail.com)

K.S. Vishal, Student, Computer Science Engineering, SRM Institute of Science and Technology, Tamil Nadu, India. (e-mail: vishalshyam@gmail.com)
}

sent to normal person's device and will also be displayed on LCD.

There are many individuals who are completely deaf and blind. For such individuals, their primary means of communication have to do with Braille or they use some other system such as tactile sign language, where they are actually in physical contact with the person they are communicating with.

\section{SYSTEM FUNCTIONALITY}

This framework utilizes a small convenient gadget that helps them to speak with abled individuals. The gadget has a QWERTY console with a LCD display on one side, and a three-cell braille display on the opposite side. The person with good sight types using the QWERTY keyboard. A deaf-blind person is able to read the printed text by placing their fingers on the Braille display. Their reply is made using the Braille input keys. The sighted person can read the text on the LCD display [3].

\section{For blind}

Firstly, for visually impaired individuals it is hard to take notes and read books like typical individuals. This paper creates and plans a portable device by which the visually impaired will probably read the letters in order one by one with the assistance of Braille characters which are in $2 * 3$ Matrix format. The input to the Braille device can be given remotely via any gadget through Bluetooth.

In this paper, servo motors (SG-90) are being utilized as buttons for engraving the Braille characters in $2 * 3$ grids as shown in the Fig 1.1. The input to the Braille device is shown with a time delay between each character. Along these lines, all the reading material to the visually impaired individuals can be given in Braille Language remotely [4]. For remote connectivity, a Bluetooth Transceiver HC-05 is used.

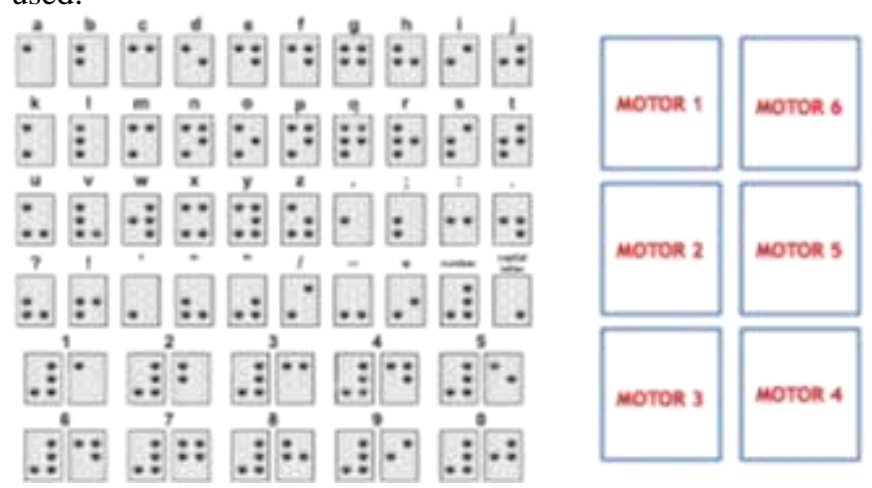

Fig. 1.1: Braille for English 


\section{For Dumb}

For mute or dumb people, input is detected using the camera. The camera uses an algorithm to understand the various gestures and convert them into text as well as into audio sounds. Like this, the different hand gestures patterns are recognized.

\section{For Deaf}

If we need to pass on something to a hard of hearing individual, the information is given by means of Bluetooth Device which has pre-loaded messages that can be shown on the LCD screen present before the individual.

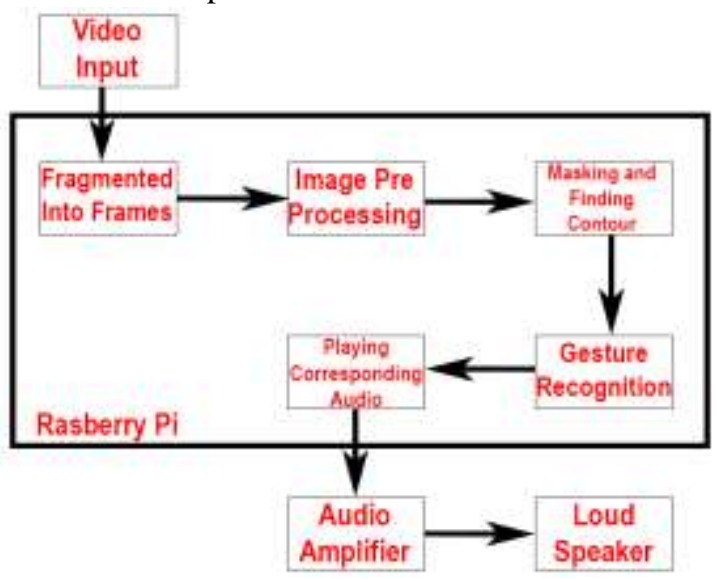

Fig. 2.1: Gesture Recognition

\section{THE TWO UNIT PORTABLE AID}

The thought is a two-unit framework intended for face to face and wireless communication between a visually disabled individual and an abled person [5]. It is operated on battery and it is portable. The deaf-blind person would utilize a 'Braille unit' with a Braille console and a three-cell (tactile) display. The abled person would utilize an 'normal unit' with a liquid crystal display (LCD). The framework can be summed up to be used as a communication portable device between two deaf-blind people as well. communication between two deaf-blind individuals would include two Braille units or the sharing of one Braille unit [6].

\section{Normal Unit}

The sighted person operates the 'normal unit' which contains the following modules:

(i) KEYBOARD: It is a QWERTY keyboard to type in the ASCII characters and the mode button is used to tell the device what type of output is to be performed as shown in Fig 3.1.

(ii) LCD DISPLAY: The LCD can display 128 characters of size $8 * 8$. The entire screen is divided into two sections: the left half for showing the message being composed in and the right half for showing the message. Port0 (POO-P07) of an Intel 8952 is used to create the corresponding English phonetic images. Pins P23 and P24 of the Intel 8952 are associated with Pins CS1 and CS2 of the DG12864 LCD. At the point when the output of the Pin P23 024 is at a highvoltage level $(+5 \mathrm{~V})$ the left (right) segment of the LCD is shows the message and vice versa. person when a normal person is trying to communicate with (iii) BUZZER: A buzzer is used to alert the disabled

them [7]. A buzzing sound and vibration indicates an incoming call by an abled person.

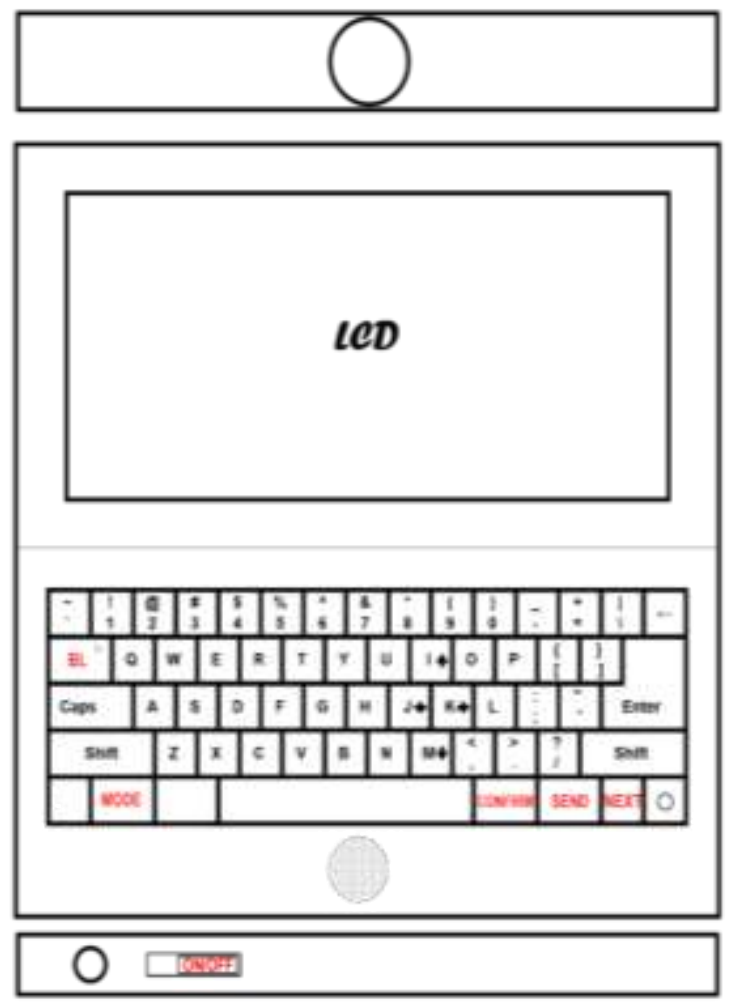

Fig. 3.1: Normal Unit

(iv) COMMUNICATION: There are two operating modes in the communication module. For wireless communication, a transmitter and a receiver are required for each unit. We divide the six-dot Braille cell into left side and right side. Each side contains three dots, in this manner, three bits are required. in addition to the three bits relating to the data of which the dot is set or cleared, another piece is required to demonstrate which side (left or right) is being transmitted. Digit 1 of the first piece demonstrates that the 3-bit information of the left half of a Braille code is being transmitted and digit 0 shows that the 3-bit information of the right side of the Braille code is being transmitted. The binary digits 1 and 0 on the other hand change to inform the receiver to correctly combine corresponding information. the HT-12E (transmitter) and the HT-12D (receiver) are utilized for remote communication.

(v) AUXILIARY PORTION: The camera is present on the back side of the normal unit. The video recording system has a reach of about $1024 \times 768$ resolution. In this project, we are using a camera which is used to capture the image and gesture control function.

(vi) OPTICAL CHARACTER RECOGNITION (OCR) USING PYTHON TESSERACT.

The text to speech algorithm is implemented with the help of Optical Character Recognition (OCR) software which is from PYTHON TESSERACT. The page and audio control is provided by Raspberry Pi model.

The program is simulated mistreatment Python code which provides the audio signal as an output. This enables the visually impaired people to read the text easily.

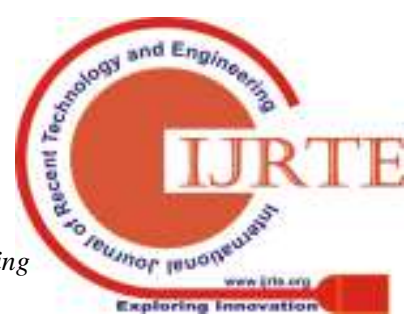




\section{Braille Unit}

The Braille unit comprises of five modules: a Braille terminal module, a Braille (material) display module, a vibrator module, a communication and a microprocessor control module.

(I) BRAILLE TERMINAL: The Braille terminal design is shown in Fig 3.2. As in the keyboard of the normal unit, there are a total of 12 keys in the terminal: six keys relating to the six spots in a Braille code and six function keys.

(ii) BRAILLE DISPLAY: There are in total 21 consonants, 5 vowels. Each character is related with one of five unique tones. Each character requires at most three cells relating to a consonant, a vowel or a diphthong, and a tone, individually. In this way, the base necessity for the Braille (material) display is three cells. As an exchange off between the complex nature of the framework and the productivity, we chose to adapt three six dot Braille cells.

(iii) COMMUNICATION: The braille device first pairs with the normal unit to establish connection. Then they can communicate using the keys provided in their devices

(iv) MICROPROCESSOR CONTROL: The Intel 8952's Port1 is used to detect which key is pressed. Six pins of Port0 (PO0 P05), Port2 (P20 P25), and Port3 (P30 P35) are utilized for three Braille cells, separately. In the meantime, Port3 is likewise used to transmit and get information by means of the RF module or phone line.

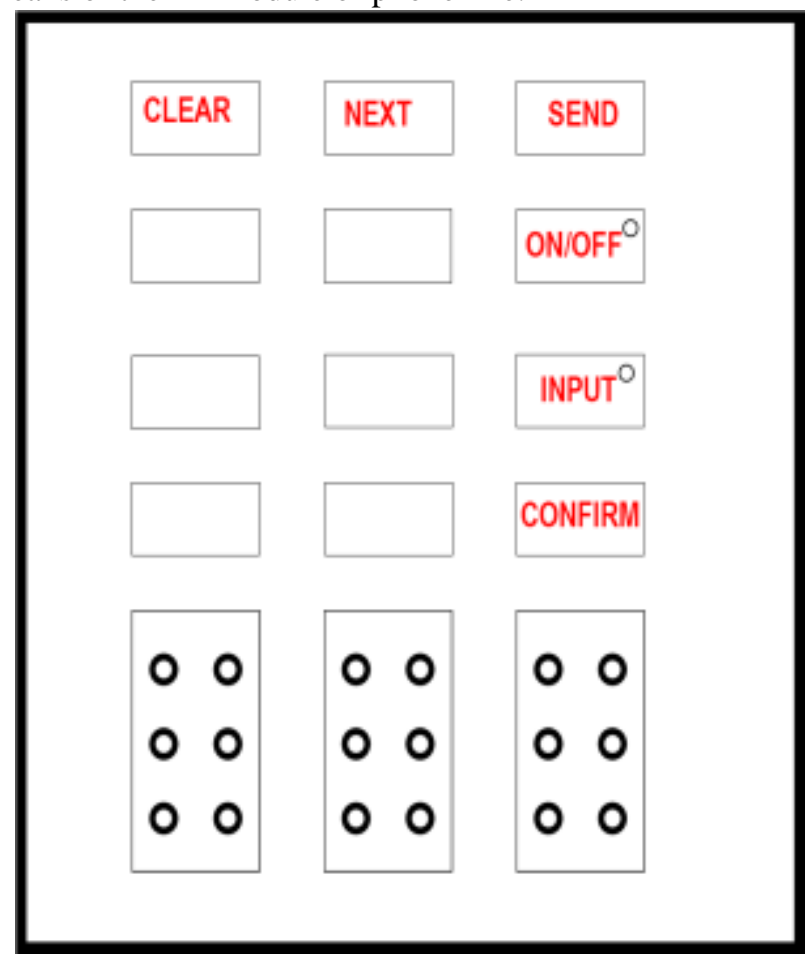

Fig. 3.2: Braille Unit

(v) VIBRATOR MODULE: The vibrator module is used to indicate the user of the normal device that the message has been received from braille device or has successfully converted the audio to text, text to audio, video to text and video to audio and also to indicate that message has been successfully received by the braille device. The standard means of producing a vibrating output in devices for the deaf and deaf-blind is to drive a small electric motor with an eccentric, out of balance weight on its shaft. A 5V DC motor of $13 \mathrm{~mm}$ diameter is selected.

\section{OPERATING MODEL \& RESULTS}

\section{Sighted-to-Disabled Mode}

In this mode, a sighted person tries to communicate with a hard of hearing, visually impaired individual. The working system includes:

The sharp-sighted person presses the 'ON/OFF' slider key and afterward checks whether the green LED is on. The green LED was incorporated into the unit to facilitate framework testing.

For wireless communication, the abled partner should press the 'BT' key to control the RF modules and check if the red diode is on. Else, it implies we should choose the face-to-communication.

When combined up with a Braille device, the sighted partner may then type in the Braille codes of the message to be transmitted. The relating English phonetic images will be shown on the LCD so the sharp-sighted individual can check if there is any typing slip-up. At whatever point a misstep happens, the sighted individual can press the 'clear' button and afterward key in the right Braille codes. Note that the 'next' key ought to be pressed at whatever point a total sixdot Braille code is entered in (a consonant, a vowel, a diphthong or a tone). At that point the 'ENTER' key ought to be pressed, when the user finishes the codes for a complete English character. After all Braille codes have been entered in, the 'SEND' key ought to be pressed to transmit those Braille codes to the Braille unit.

The Braille codes square measure transmitted Bluetooth signal. At that point these Braille codes will be shown on the three-cell Braille (tactile) display with the goal that the hard of hearing visually impaired person can read the message. ,After the deaf-blind partner reads the message, the 'CONFIRM' key should be pressed to inform the sighted partner to indicate that the message has been received. In the meantime, all language phonetic images vanish from the LCD display. The sighted individual may wait for the reaction from the deaf-blind individual or keep keying another message.

\section{Disabled-to-Sighted Mode}

In this mode, deaf-blind individual tries to speak with a sighted individual [8].

Press the 'ON/OFF' key and check whether the vibrator creates an uncommon rhythmical vibration. The 'Input' key is utilized for flipping among sending and accepting modes.

The deaf-blind individual may enter in the Braille codes of the message to be transmitted. Note that these Braille codes will be shown on the three-cell Braille (tactile) display with the goal that the deaf-blind individual can check whether there is an error, the user can press the 'CLEAR' key to clear the Braille codes and re-enter them. The 'NEXT' key ought to be pressed whenever a total six-spot Braille code is entered in. After all Braille codes have been entered in, the 'SEND' key should be pressed to transmit the message to the normal unit. 
The 'CONFIRM' key is used to tell the other individual that they have effectively gotten their data or to tell the other individual that they have completed their message that will be passed on.

When the Braille codes are transmitted by means of the RF modules or phone line, the three six-dot Braille cells are then all set to see the user the message were transmitted. The Braille codes will be converted into their corresponding English phonetic images and showed on the LCD displayed [9]. At the point when all dots are cleared, the deaf-blind individual may wait for the reaction from the sighted individual or keep keying new messages.

\section{Between Two Sighted Persons}

1) This mode characterizes the communication between two sighted people with difficulties (deaf-dumb). A dumb person can communicate with a deaf individual via gestures or by messaging to the device. The device makes an interpretation of gesture into content format which will be shown on LCD screen. Through LCD screen the deaf individual could comprehend what the other individual is trying to convey.

\section{a. Optical Character Recognition (OCR) Using Python TESSERACT}

The text to speech algorithm is implemented with the help of Optical Character Recognition (OCR) software which is from PYTHON TESSERACT. The page and audio control is provided by Raspberry $\mathrm{Pi}$.

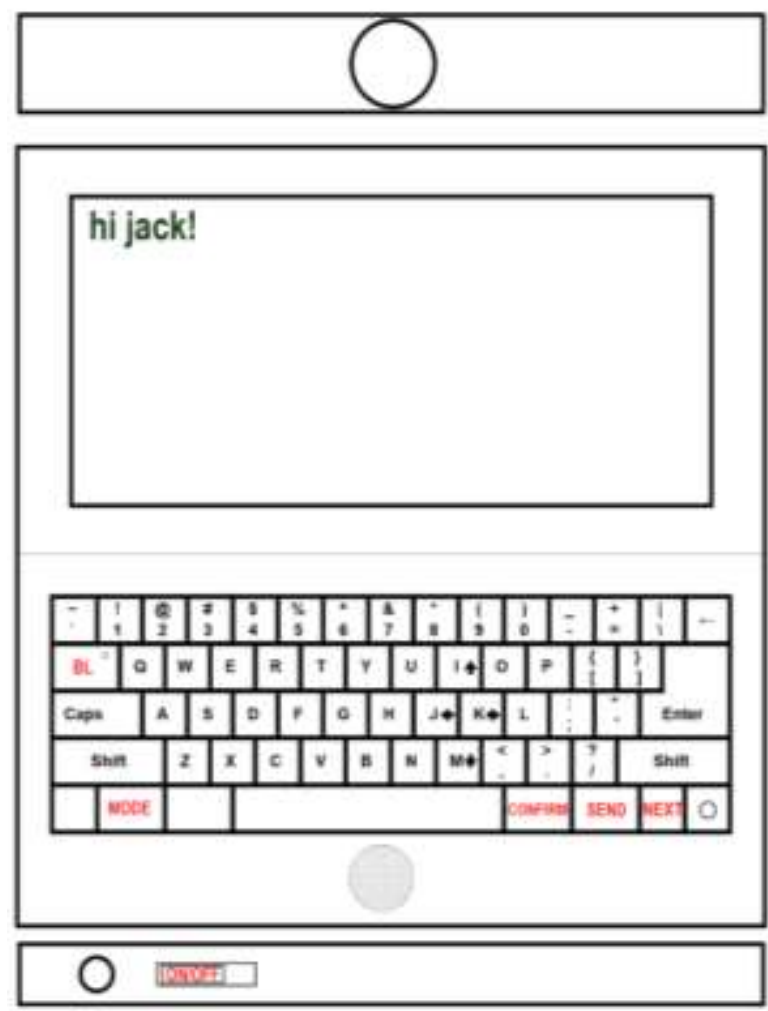

The program is simulated mistreatment Python code which provides the audio signal or in text form as an output. This enables the other person to read the message or hear the message easily.

2). If a deaf person needs to respond, the person can do so by speaking into the device which gets converted into text and is displayed on the LCD screen.

\section{CONCLUSION}

Deaf, dumb and blind people have difficulties in interacting with the world, so they need assistance of somebody. This model enables them to have an increasingly normal life and be progressively self-sufficient. This paper effectively produces a portable device to expel the hindrance of communication between deaf-dumb \& blind. As a result, the system is connective to the user's social, emotional and cognitive development .

\section{FUTURE SCOPE \& RESULTS}

The system can be modified to connect to the internet for communication over long distance. By connecting to a server which holds various datasets for gestures, the device's gesture recognition can be improved. We can employ various vibration levels such as weak vibrations and strong vibrations for indicating various messages and notification to improve efficiency for disabled people.

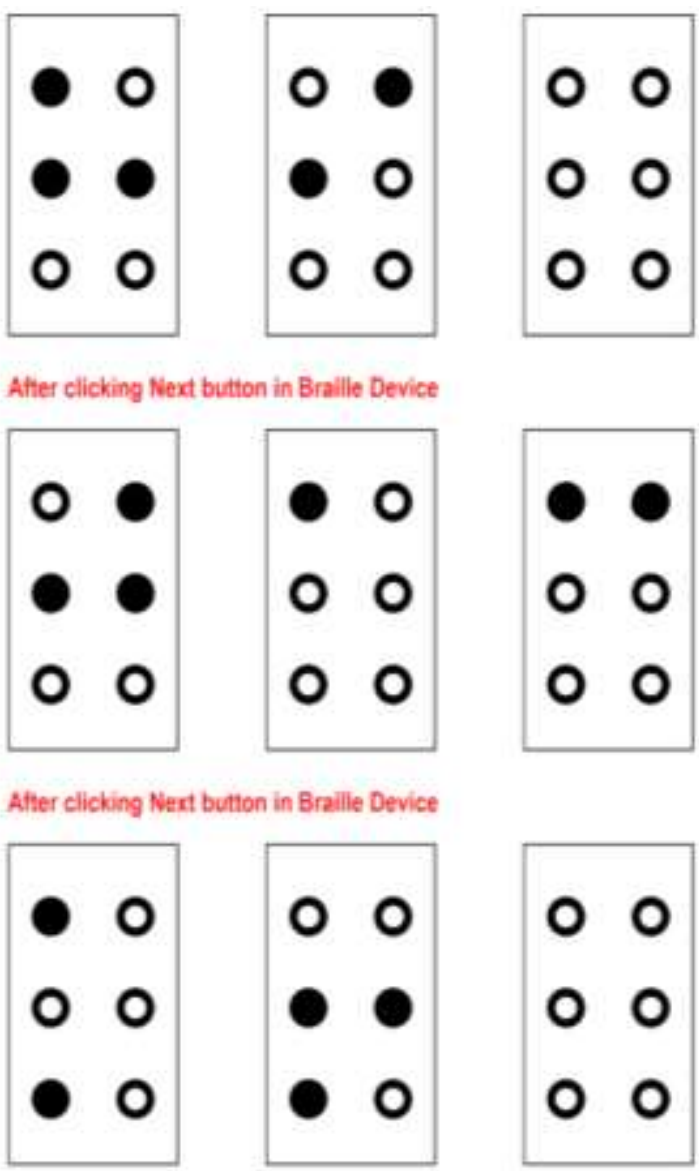

Fig. 4: Experimental Outcome 


\section{REFERENCES}

1. Telebraille Communication System Product Brochure 1987: Telesensory Systems, Inc., 455 North Bernardo Avenue, Mountain View, CA 94039, USA

2. SZETO, A. Y. J., and SAUNDERS, E A.: 'Electrocutaneous stimulation for sensory communication in rehabilitation engineering', IEEE Trans. Biomed. Engineering, 1982, 29, (4), pp.300308

3. How do Deaf-Blind People Communicate? Available at: http://www.aadb.org/factsheets/db_communications.h tml.

4. A Novel Approach to Communicate with Deaf Dumb and Blind Person - International Journal of Engineering Research \& Science (IJOER) ISSN: [2395-6992] [Vol-3, Issue-4, April- 2017]

5. LKJ, L. T., and BALACHANDRAN, W.: 'Electronic compass for blind or deaf-blind pedestrians', Electronics Letters, 1993,29, pp.1462-1463

6. BAZZANI, M., and MUMOLO, E. M.: 'PC-based telephone communication system for deaf-blind people', IEEE Global Telecommunication conference, 1998, pp.43-47

7. DAMPER, R. I., and ERANS, M. D.: 'A multifunction domestic alert system for the deafblind', IEEE Trans on Rehabilitation Engineering, 1955,3, (4), pp.354-359

8. SZETO, A. Y. J., and CHRISTIANSEN, K. M.: 'Technological device for deaf-blinded children: need and potential impact', IEEE Engineering in Medicine and Biology Magazine, September 1988, pp.25-29

9. Tact aid I1 Product Brochure 1987: Audiological Engineering Corporation, 9 Preston Road, Sommervilla, MA 02143, USA 